\title{
PERCEPÇÕES DOS IDOSOS SOBRE PLANTAS TÓXICAS
}

Bruna Cristina Mendes dos Santos ${ }^{1}$, Leonardo Teixeira Ramoniga ${ }^{2}$, Andréia Assamy Guinoza $^{3}$, Mirian Ueda Yamaguchi ${ }^{4}$, Lúcia Elaine Ranieri Cortez ${ }^{5}$.

1- Graduanda em Medicina no Centro Universitário Cesumar - UniCesumar, Maringá, Paraná, Brasil (bcmsantos2008@hotmail.com).

2- Graduando em Medicina no Centro Universitário Cesumar - UniCesumar, Maringá, Paraná, Brasil

3- Docente do Curso de Estética e Cosmética do Centro Universitário Cesumar UniCesumar, Paraná, Brasil

4- $\quad$ Docente do Curso de Mestrado em Promoção da Saúde do Centro Universitário Cesumar - UniCesumar, Paraná, Brasil

5- $\quad$ Docente do Curso de Mestrado em Promoção da Saúde e do Curso de Medicina do Centro Universitário Cesumar - UniCesumar, Paraná, Brasil

Recebido em: 08/09/2015 - Aprovado em: 14/11/2015 - Publicado em: 01/12/2015 DOI: http://dx.doi.org/10.18677/Enciclopedia_Biosfera_2015_261

\section{RESUMO}

As plantas tóxicas provocam inúmeros casos de intoxicação, principalmente pelo fato de serem usadas como plantas ornamentais e pela desinformação sobre a ação destas. Os idosos representam um grupo que faz uso difundido destas plantas e ainda há a necessidade de estudos sobre o conhecimento destes em relação às plantas. Desta forma, objetivou-se verificar o conhecimento dos idosos sobre plantas tóxicas. Foram aplicados questionários contendo informações sócio-demográficas, bem como, cuidados e conhecimento popular sobre plantas tóxicas. Para verificar possíveis associações entre as variáveis de interesse foram aplicados o Teste Quiquadrado. A maioria dos idosos não relatou conhecimento popular sobre plantas tóxicas, no entanto, os que o fizeram, referenciaram principalmente a planta Comigoninguém-pode (Dieffenbachia picta Schott.) e com menor prevalência a Espirradeira (Nerium oleander L.) e Copo de leite (Zantedeschia aethiopica Spreng.). Quanto às intoxicações, $29,5 \%$ dos entrevistados relataram algum caso de intoxicação. Em relação aos cuidados com plantas tóxicas, $62,15 \%$ afirmaram o ter, no entanto, $68,92 \%$ relataram que cultivam essas plantas próximas de crianças e animais, no entanto, algumas plantas citadas como tóxicas pelos idosos, são utilizadas popularmente como medicinais. Conclui-se, portanto, que muitos idosos não têm conhecimento sobre plantas tóxicas. Algumas destas são utilizadas como medicinais e o cultivo das mesmas é próxima de animais e crianças. O desconhecimento com estas espécies vegetais é um dos principais fatores para ocorrência de acidentes. Medidas de promoção à saúde e prevenção acerca de intoxicações em geral podem ser fatores indispensáveis, já que a maioria das intoxicações é acidental e, portanto, evitáveis.

PALAVRAS-CHAVE: intoxicação; saúde pública; geriatria; políticas educativas. 


\title{
PERCEPTIONS OF ELDERLY ON TOXIC PLANTS
}

\begin{abstract}
Toxic plants causing numerous cases of poisoning, mainly because they are used as ornamental plants and the misinformation about the action of these. The elderly represent a group that makes widespread use of plants and there is still a need for studies on their knowledge in relation to plants. This work aimed to verify the knowledge of the elderly about toxic plants. Questionnaires were applied containing socio-demographic information, as well as care and popular knowledge about toxic plants. To verify possible associations between the variables of interest were applied the chi-square test. Most seniors reported no popular knowledge of poisonous plants, however, those who did, have referred mainly to Me-anyone-can plant (Dieffenbachia picta Schott.) And a lower prevalence Oleander (Nerium oleander L.) and Glass milk (Zantedeschia aethiopica Spreng.). As for the poisoning, 29.5\% of respondents reported a case of poisoning. Regarding the care with toxic plants, $62.15 \%$ said they have, however, $68.92 \%$ reported that cultivate these plants near children and animals, however, some plants cited as toxic by the elderly, they are popularly used as medicinal. It can be concluded therefore that many older people have no knowledge about toxic plants, some of these are used as medicinal and cultivation of them is close to animals and children. Unfamiliarity with these plant species is one of the main factors for the occurrence accidents. Promotion of health and prevention measures about general poisoning can be essential factors, since most poisonings are accidental and therefore preventable.
\end{abstract}

KEYWORDS: elderly; intoxication; toxic plants.

\section{INTRODUÇÃO}

Historicamente o ser humano tem estreito contato com o meio ao seu redor e todas as civilizações que habitaram a Terra utilizaram em maior ou menor medida as plantas, sendo a razão pela qual se tem aperfeiçoado o conhecimento sobre as espécies medicinais (OTAIZA et al., 2006). Dentre as 250 mil espécies de vegetais superiores, muitas dessas são tóxicas por produzirem substâncias capazes de exercerem alterações fisiológicas nos organismos vivos e em alguns casos sérios transtornos levando o individuo a óbito (SIMÕES et al., 2010).

Muitos princípios ativos são potencialmente tóxicos para os seres vivos, e dentre as famílias de plantas há muitas espécies que se destacam por possuí-los como: Solanaceae, Apocynaceae e Euphorbiaceae, entre outras. Em análises fitoquímicas de plantas destas famílias, os compostos nitrogenados, tais como alcalóides e glicosídeos, podem implicar em risco potencial para seres vivos diversos. Dentre os princípios ativos tóxicos, destacam-se alguns cianogênicos, que se hidrolisam em ácido cianídrico e aldeído benzoico, liberando como se pode supor, traços de cianureto no organismo com consequências tóxicas (OTAIZA et al., 2006).

As substâncias tóxicas das plantas podem afetar diversos órgãos vitais, bem como o Sistema Nervoso Central (SNC) interferindo assim na coordenação das funções nervosas do corpo. As toxinas mais dominantes são neurotoxinas que afetam o cérebro e SNC, seguido por citotoxinas e toxinas metabólicas que afetam órgãos como os rins, o fígado, o coração e os pulmões. A gravidade de um efeito tóxico pode depender da via de administração, da fase de crescimento ou parte da planta, a quantidade consumida, as espécies e a susceptibilidade da vítima. A solubilidade da toxina nos fluidos corporais, a frequência de intoxicação, bem como 
a idade da vitima, também são fatores que podem influenciar na toxicidade das plantas (NDHLALA et al., 2013).

A maioria das plantas tóxicas mais comuns são cultivadas em jardins como plantas ornamentais (ALBURQUERQUE, 1980). Sabe-se que tanto plantas medicinais como as ornamentais podem ser potencialmente tóxicas, e dependem somente da dose utilizada para atingirem esse potencial, deve-se, portanto saber os limites bioquímicos entre a terapêutica e a toxicidade (OTAIZA et al., 2006).

A ocorrência de acidentes com essas plantas muitas vezes não são evitados, uma vez que, essas informações sobre a toxicidade destas não chegam à população e nem aos profissionais da área da saúde, aumentando o risco em casos de ingestão quando o atendimento não é rápido e eficiente (OLIVEIRA et al., 2006). Dentre os fatores que dificultam o diagnóstico, tal como o tratamento dessas intoxicações, segundo PINILLOS et al. (2003), está ligado a desinformação da população, a cultura popular, e a quantidade ingerida pelo paciente.

Muitos problemas surgem das consequências da elevada incidência de intoxicações agudas, portanto há a necessidade de encontrar uma solução para estes problemas. Desta forma a Organização Mundial da Saúde, assim como a Organização Panamericana da Saúde tem incentivado a implementação e o desenvolvimento de um serviço de informação toxicológica, que seja de alcance tantos dos profissionais da saúde quanto para a população em geral, favorecendo uma melhor qualidade de vida (PEACOK et al., 2009), já que o conhecimento do potencial tóxico de algumas espécies de plantas são importantes no sentido de prevenir possíveis efeitos indesejáveis.

De acordo com, JUNIOR et al. (2013) o uso de plantas por idosos é bastante difundido. Isso demostra a importância de estudos que busquem caracterizar as percepções desse grupo específico relacionado a essas plantas.

Diante do exposto, objetivou-se verificar o conhecimento dos idosos sobre plantas tóxicas.

\section{Caracterização da área de estudo:}

\section{MATERIAL E MÉTODOS}

O local de estudo foi o CISAMUSEP, um consórcio público de saúde que presta serviços para os 30 municípios do AMUSEP (Associação dos Municípios do Setentrião Paranaense). Esse disponibiliza consultas especializadas e procedimentos, com organização, informatização e treinamento para as equipes de atendimento de saúde desses municípios, além de buscar soluções conjuntas para enormes problemas de saúde (CISAMUSEP, 2014).

\section{Participantes:}

Os participantes desse estudo foram os idosos frequentadores do CISAMUSEP (Maringá - PR) com idade igual ou maior que 60 anos. Foram excluídos do estudo idosos com demência, quadro clínico instável, com flutuações cognitivas e do nível de consciência.

O trabalho foi regulamentado pelas normas éticas para pesquisa envolvendo seres humanos. A pesquisa foi aprovada pelo Comitê de Ética em Pesquisa (CEP) do Centro Universitário de Maringá (UniCesumar) sob parecer $144.818 \mathrm{em}$ 13/11/2012. A participação dos idosos ocorreu mediante a assinatura do Termo de Consentimento Livre e Esclarecido (TCLE) obedecendo às recomendações da Resolução nº 196/96, da Comissão Nacional de Saúde. 


\section{Cálculo da amostra:}

A amostra foi calculada assumindo nível de confiança de 95\%, margem de erro de $5 \%$, e com uma população estimada de aproximadamente 4.527 indivíduos. Esse número representa o total de pacientes considerados na pesquisa realizada no período de três meses, a amostra foi composta por 251 idosos com idade superior a 60 anos.

\section{Coleta de dados:}

O levantamento das informações foi do tipo quantitativo, exploratório, descritivo e de campo, e os 251 questionários aplicados continham questões semiestruturadas, contendo informações sócio demográficas, bem como, cuidados e conhecimento sobre plantas tóxicas.

Foram calculadas as médias e os desvios padrão para as variáveis quantitativas, e organizadas por meio de tabelas de frequência. Para verificar possíveis associações significativas entre as variáveis de interesse foram aplicados - Teste Qui-quadrado e o Teste Exato de Fischer. O nível de significância adotado foi de $5 \%$, ou seja, foram consideradas significativas as associações cujo $p<0,05$.

\section{RESULTADOS E DISCUSSÃO}

Observa-se na Tabela 1 que a maioria $(71,31 \%)$ dos idosos participantes da pesquisa pertencia ao sexo feminino. No entanto, quando se avalia o sexo dos idosos que afirmam saber sobre a existência de plantas tóxicas, não há diferença entre os gêneros $(p=0,0637)$, apesar do número de idosos do sexo feminino ser maior $(78,13 \% \mathrm{n}=75)$.

Quanto à idade dos idosos entrevistados $66 \%(n=166)$ estavam na faixa de 60 a 69 anos. Segundo o SINITOX (2009), o número de envenenamento por plantas tóxicas ocorre mais com mulheres na faixa etária de 60 a 69 anos, em concordância com os dados da pesquisa. Em relação à escolaridade $94(62,6 \%)$ pessoas que sabem da existência de plantas tóxicas nunca foram à escola ou fizeram o primário incompleto e $71,61 \%$ ( $n=111)$ dos idosos que cultivam plantas tóxicas ganham mais de um salário mínimo por mês.

Tabela 1. Características sociodemográficas dos idosos frequentadores do CISAMUSEP, que participaram da pesquisa sobre plantas tóxicas.

\begin{tabular}{|c|c|c|c|c|c|}
\hline \multirow{3}{*}{ Características } & \multicolumn{4}{|c|}{ Tem conhecimento } & \multirow{3}{*}{$p^{*}$} \\
\hline & \multicolumn{2}{|c|}{ Sim } & \multicolumn{2}{|l|}{ Não } & \\
\hline & Quant. & $\%$ & Quant. & $\%$ & \\
\hline \multicolumn{5}{|l|}{ Gênero } & \multirow{4}{*}{0,0637} \\
\hline Feminino & 75 & 78,13 & 104 & 67,10 & \\
\hline Masculino & 21 & 21,88 & 51 & 32,90 & \\
\hline \multicolumn{5}{|l|}{ Idade } & \\
\hline 60 a 69 anos & 68 & 70,83 & 98 & 63,23 & \multirow{3}{*}{0,3280} \\
\hline 70 a 79 anos & 22 & 22,92 & 49 & 31,61 & \\
\hline $\begin{array}{l}80 \text { a } 89 \text { anos } \\
\text { Escolaridade }\end{array}$ & 6 & 6,25 & 8 & 5,16 & \\
\hline Nunca foi à escola & 35 & 23,33 & 25 & 26,88 & \\
\hline Primário incompleto & 59 & 39,33 & 35 & 37,63 & 0,9269 \\
\hline Primário completo & 34 & 22,67 & 21 & 22,58 & \\
\hline 10 grau completo ou mais & 22 & 14,67 & 12 & 12,90 & \\
\hline \multicolumn{5}{|l|}{ Renda } & \\
\hline abaixo de 1 salário & 8 & 5,16 & 3 & 3,13 & \multirow{3}{*}{0,3896} \\
\hline 1 salário & 36 & 23,23 & 29 & 30,21 & \\
\hline Acima de 1 salário & 111 & 71,61 & 64 & 66,67 & \\
\hline
\end{tabular}

ENCICLOPÉDIA BIOSFERA, Centro Científico Conhecer - Goiânia, v.11 n.22; p.3740 2015 
Dentre as plantas tóxicas mais citadas pelos idosos (Tabela 2) destaca-se a Comigo-ninguém-pode (Dieffenbachia picta Schott.) (89\%), que está entre as principais plantas com características toxicológicas citadas pelo SINITOX (2009). Entre as referidas no estudo com menor prevalência estão: Leiteiro da Amazônia (Sinadenium clarinatum Boiss) (3\%), Copo de leite (Zantedeschia aethiopica Spreng.) (2\%), Espirradeira (Nerium oleander L.) (1\%), Arruda (Ruta graveolens L.) (1\%), Guiné (Petiveria alliacea L.) (1\%).

Pode ser observado que algumas plantas citadas como tóxicas são utilizadas com plantas medicinais como é o caso do Guiné (Petiveria alliacea L.). P. alliacea $L$. apresenta efeitos antiinflamatórios, anti-helmíntico, antimicrobianos e antineoplásicos, sendo muito utilizada na América do Sul e Central (KUBEC \& MUSAH, 2002; CAMARGO, 2007). GUEDES et al. (2009) também demonstraram atividade antimicrobiana em estudo com extratos de guiné, visto que os mesmos apresentaram inibição do crescimento das bactérias Staphylococcus aureus e Enterococcus faecalis, bem como, a levedura Candida albicans. Mas o uso medicinal deve ser controlado, pois, o Guiné apresenta atividade tóxica subaguda em dose de $1270 \mathrm{mg} / \mathrm{kg}$ de seu extrato hidroalcoólico das folhas e das raízes, podendo apresentar características abortivas, citotóxica, zigotóxicas e antimitótica (DI STASI \& HIRUMA-LIMA, 2002).

O Leiteiro da Amazônia ou Janaúba, também é utilizado popularmente como planta medicinal, seu uso tradicional é feito com a diluição do látex da planta em água pura e fresca - 18 gotas de látex em um litro de água. Suas aplicações foram bastante difundidas popularmente para a cura de diversos tipos de câncer e para outras enfermidades com úlceras e inflamações, mas são poucos estudos a respeito das supostas ações farmacológicas da planta, não havendo, portanto, evidências científicas que as comprovem. Já seus metabólitos secundários tem ação analgésica, antiinflamatória, citotóxica, antimicrobiana, antiviral, fungicida e inseticida. Contudo, essa planta apresenta glicosídeos cianogênicos ou cianogenéticos, que são potencialmente tóxicos para um grande número de organismos vivos, demonstrando o risco da administração dos extratos dessa planta em altas concentrações (OLIVEIRA et al., 2006).

A arruda (Ruta graveolens L.) é usada com frequência como planta medicinal, suas principais propriedades se dão pela substância rutina, ela é usada para aumentar a resistência dos vasos sanguíneos, evitando rupturas e, por isso é indicada no tratamento contra varizes (BLANCO, 2010). O chá de suas folhas pode ser usado contra asma, pneumonia, dor de cabeça, analgésico, antiespasmódico, tranquilizantes e problemas uterinos. O suco de suas folhas é usado como abortivo e contra acidente vascular encefálico, suas folhas também podem ser usadas contra dismenorreia, diarreias e febre, enquanto o xarope das suas folhas é usado contra tosses graves. Além disso, suas sementes servem como anti-helmínticos e parasiticidas. Contudo, há relatos de que $R$. graveolus pode provocar dermatites devido a um mecanismo fototóxico que torna a pele sensível à luz solar induzindo dermatites e também dores epigástricas, cólicas, vômitos, arrefecimento da pele, depressão do pulso, contração das pupilas, convulsões e sonolência após a sua ingestão (DI STASI \& HIRUMA-LIMA, 2002). 
Tabela 2- Principais plantas tóxicas citadas pelo SINITOX (2009) e idosos.

\begin{tabular}{|c|c|c|c|}
\hline Nome Popular & Nome Científico & SINITOX & Idosos \\
\hline Tinhorão & Caladium bicolor Vent. & Citada & Não citada \\
\hline Comigo-ninguém-pode & Dieffenbachia picta Schott. & Citada & Citada \\
\hline Taioba-brava & Colocasia antiquorum Schott. & Citada & Não citada \\
\hline Copo-de-leite & $\begin{array}{c}\text { Zantede } \\
\text { schiaaethiopica Sprena. }\end{array}$ & Citada & Citada \\
\hline Saia-branca & Datura suaveolens L. & Citada & Não citada \\
\hline Aroeira & Lithraea brasiliens March. & Citada & Não citada \\
\hline Bico-de-papagaio & Euphorbia pulcherrima Willd. & Citada & Não citada \\
\hline Coroa-de-cristo & Euphorbia milii L. & Citada & Não citada \\
\hline Avelós & Euphorbia tirucalli L. & Citada & Não citada \\
\hline Urtiga & Fleurya aestuans L. & Citada & Não citada \\
\hline Espirradeira & Nerium oleander L. & Citada & Citada \\
\hline Chápeu-de-napoleão & Thevetia peruviana Schum. & Citada & Não citada \\
\hline Cinamomo & Melia azedarach L. & Citada & Não citada \\
\hline Mandioca-brava & Manihot utilissima Pohl. & Citada & Não citada \\
\hline Mamona & Ricinus communis $\mathrm{L}$. & Citada & Citada \\
\hline Pinhão-roxo & Jatropha curcas L. & Citada & Não citada \\
\hline Leiteiro da Amazônia & Sinadenium clarinatum & Não citada & Citada \\
\hline Arruda & Ruta graveolens L. & Não & Citada \\
\hline Guiné & Petiveria alliacea $L$. & Não citada & Citada \\
\hline Banana de macaco & Philodendron bipinnatifidum & Não citada & Citada \\
\hline
\end{tabular}

A Tabela 3 apresenta o relato dos idosos quanto à ocorrência de sintomas de intoxicação, sendo que dentre estes 29,5\% ( $n=74)$ o afirmaram , sendo a ocorrência nos mesmos ou em outra pessoa. Entre os idosos entrevistados, a maioria (67\%) citou a Comigo-ninguém-pode como a causadora das atividades tóxicas, resultados semelhantes foram obtidos em estudo realizado em Belém/PA onde a mesma foi a mais citada nos casos de intoxicação (VASCONCELOS et al., 2009). Os sintomas de intoxicação relatados devido a esta planta foram: vômito, sialorreia, prurido, eritema, feridas, diarreia, edema, dispneia, tosse e síncope. Segundo SINITOX (2009) os principais sintomas causados pela intoxicação da Comigo-ninguém são dor em queimação, eritema e edema de lábios, língua, palato e faringe, sialorréia, disfagia, asfixia, cólicas abdominais, náuseas, vômitos e diarreia e quando há contato ocular ocorre irritação intensa com congestão, edema, fotofobia e lacrimejamento. Pode-se observar que de um modo geral os sintomas relatados nos casos conhecidos de intoxicação segundo os idosos, se assemelham aos principais sintomas tóxicos da planta.

A Banana de macaco (Philodendron bipinnatifidum Schott ex Endl) e a mamona (Ricinus communis L.) também foram citadas como causadoras de intoxicações, e estão incluídas na lista de principais plantas tóxicas do SINITOX (2009). Contudo, algumas plantas foram citadas como tóxicas por apenas um idoso em cada caso (tabela 2), mas são utilizadas na maioria das vezes como plantas medicinais sendo Acerola (Malpighia emarginata DC.), Quebra-pedra (Phyllanthus niruri L.), Boldo (Peumus boldus Molina), Tanchagem (Plantago major L.) e Café (Coffea arabica L.).

O boldo (Peumus boldus Molina) é uma das plantas medicinais mais utilizadas popularmente, e suas folhas são tradicionalmente empregadas em distúrbios hepáticos. Quimicamente apresenta boldina, flavonóide e catequinas 
(OCHOA et al., 2008; YU et al., 2009) que tem demonstrado alguns benefícios, como a ação antiinflamatória e antioxidante (BRIAN et al 2006). Além disso, o óleo essencial da $P$. boldus, possui atividade antibacteriana contra Streptococcus pyogenes, Micrococcus sp., Sthaphylococcus aureus, Bacilus subtilis (VILA et al., 1999) e antifúngica contra diversas espécies de Cândida (LIMA et al., 2006). MEJÍADOLORES et al. (2014), evidenciaram um efeito neurotóxico do extrato aquoso de boldo em ratos após exposição prolongada. Em outros estudos foram relatados indícios de hepatotoxicidade, devendo ser consumido com moderação (RUIZ et al., 2008). Além disso, o extrato hidroetanólico das folhas de $P$. boldus mostrou efeito abortivo e teratogênico em ratos (ALMEIDA et al., 2000) e um caso de dermatite alérgica ocupacional foi relatado por manejo do boldo por um farmacêutico (GIELEN \& GOOSSENS, 2001), bem como, um caso de reação anafilática foi descrito por MONZÓN et al., (2004), em um homem com histórico de rinoconjuntivite alérgica a pólen, após a ingestão do chá de boldo. Dessa forma, mesmo que o $P$. boldus apresente ações benéficas, deve ser usado com cautela, pois pode levar a reações adversas dependendo da dose de uso, tempo de exposição e sensibilidade de cada organismo a seus componentes.

A quebra-pedra (Phyllanthus niruri L.) e a tanchagem (Plantago major L.) também estão entre as plantas medicinais mais usadas popularmente. $P$. niruri é utilizada no Brasil e em outras partes do mundo, a sua utilização bloqueia a formação de cristais de oxalato de cálcio e a formação de pedra na urolitíase (ASARE et al., 2012). Já a tanchagem, é usada em gargarejos contra inflamações de garganta, anginas e gengivas sangrentas. Faz-se o uso também para inflamações bucofaríngeas, dérmicas, gastrointestinais e das vias urinárias. Além disso, é empregada como expectorante, antidiarreica, cicatrizante, adstringente, emoliente e depurativa do sangue (LORENZI \& MATOS, 2002).

Tabela 3 - Relação das ocorrências de sintomas por plantas tóxicas citadas pelos idosos

\begin{tabular}{|c|c|c|c|c|}
\hline \multicolumn{5}{|c|}{ Número de pessoas que conhecem casos de intoxicação } \\
\hline \multicolumn{3}{|c|}{ Sim } & \multicolumn{2}{|c|}{ Não } \\
\hline $\begin{array}{c}\text { Idosos } \\
74\end{array}$ & \multicolumn{2}{|c|}{$\begin{array}{c}\% \\
29,50\end{array}$} & $\begin{array}{c}\text { Idosos } \\
177\end{array}$ & $\begin{array}{c}\% \\
7050\end{array}$ \\
\hline \multicolumn{5}{|c|}{ Plantas citadas que provocaram intoxicação } \\
\hline Nome & $\mathrm{n}$ & $\%$ & & \\
\hline $\mathrm{CNP}^{*}$ & 59 & $29,50 \%$ & Vômito, diarrei & eritema, tosse \\
\hline Jasmin & 1 & $1,15 \%$ & Vôr & reia \\
\hline Banana de macaco & 1 & $1,15 \%$ & Feri & orpo \\
\hline Folha de acerola & 1 & $1,15 \%$ & & \\
\hline Quebra pedra & 1 & $1,15 \%$ & & \\
\hline Café & 1 & $1,15 \%$ & Cef & ema \\
\hline Boldo & 1 & $1,15 \%$ & Dor & nais \\
\hline Mamona & 1 & $1,15 \%$ & & reia \\
\hline Tanchagem & 1 & $1.15 \%$ & & \\
\hline Não lembra & 21 & $23,80 \%$ & & \\
\hline
\end{tabular}

${ }^{*}$ Comigo-ninguém-pode

Analisando a figura 1, a maioria dos idosos $(62,15 \%)$ relatou conhecimento dos cuidados com plantas tóxicas, dado este importante, visto que grande parte dos 
casos de intoxicação é acidental ou por uso indiscriminado de plantas medicinais potencialmente tóxicas (RODRIGUES \& GUEDES, 2006).

Em 2012 foram registrados pelo SINITOX (2015) 113 casos de intoxicação de animais por plantas tóxicas e dos 1185 casos de intoxicação por esse agente tóxico, $60 \%$ foram com crianças de até nove anos. Destes $77 \%$ foram acidentais, já que os locais em que as pessoas realizam seus cultivos, muitas vezes é de fácil acesso a crianças e animais. Estes dados corroboram a esta pesquisa, uma vez que 68,92\% $(n=173)$ dos idosos afirmaram que as plantas tóxicas cultivadas estão próximas de crianças e animais (Figura 1). A população deve ser conscientizada sobre o risco potencial que estas plantas representam, já que elas estão constantemente presentes na vida cotidiana da população devido ao uso associado à ornamentação de diversos ambientes públicos e, também, residenciais. A nomeação das plantas nesses ambientes comunitários pode servir como uma forma de instruir a população do nome popular e científico de cada uma das espécies, assim como fornecer avisos que se tratam de plantas toxicas com potencial risco a saúde. (BOCHNER, 2006).

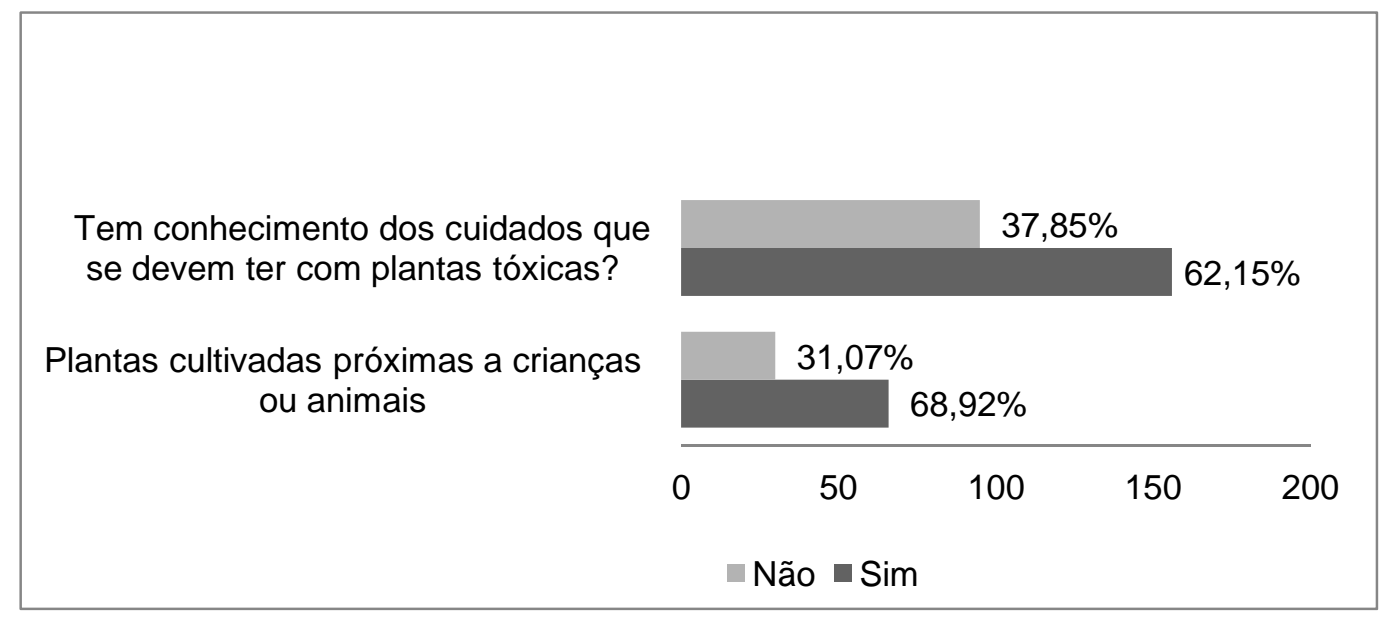

Figura 1 - Conhecimento dos idosos sobre cuidados e os locais de cultivo de plantas tóxicas.

Para ALBUQUERQUE (1980) o conhecimento sobre tal assunto deve ser expandido também para médicos e veterinários. Dados como a descrição botânica da planta, com imagem detalhada, nome popular e científico, são informações que esses profissionais devem ter como fundamentais para identificar a planta tida como tóxica e adotar o tratamento adequado, pois o tempo pode ser crucial para um melhor prognóstico nesses casos.

\section{CONCLUSÃO}

Pode-se observar o não conhecimento da maioria dos idosos em relação às plantas tóxicas, sendo que algumas destas são utilizadas como medicinais e o cultivo das mesmas é próxima de animais e crianças. O desconhecimento, ou o descuido com estas espécies vegetais é um dos principais fatores para ocorrência de acidentes. Isto indica a necessidade de se realizar trabalhos educativos e preventivos com a divulgação das espécies tóxicas junto à população, contribuindo para a diminuição dos acidentes. Medidas de promoção à saúde e prevenção acerca de intoxicações em geral podem ser fatores indispensáveis para mudar essa realidade, já que a maioria das intoxicações é acidental e, portanto, evitáveis. 


\section{REFERÊNCIAS}

ALBUQUERQUE, J.M. Plantas tóxicas no Jardim e no Campo. FCAP. Belém. 120 p. 1980.

ALMEIDA E.R.; MELO, AM.; XAVIER, H . Toxicological evaluation of the hydroalcohol extract of the dry leaves of Peumus boldus and boldine in rats. Phytotherapy Research, v. 14, n.2, p. 99-102, 2000.

ASARE, G. A.; BUGYEI, K.; SITTIEI, A; YAHAYA, E.S.; GYAN, B.; ADJEI, S.; ADDO, P.; WIREDU, E.K.; ADJEI, D.N.; NYARKO, A. K. Genotoxicity, cytotoxicity and toxicological evaluation of whole plant extracts of the medicinal plant Phyllanthus niruri (Phyllanthaceae). Genetics and Molecular Research, v.11, n. 1, p.100-111, 2012.

BLANCO, R. A. Arruda, 2010. Disponível em: <http://www.jardimdeflores.com.br/ERVAS/A05arruda.htm> Acesso em: 24 março 2015.

BOCHNER, R. Perfil das intoxicações em adolescentes no Brasil no período de 1999 a 2001. Cadernos de Saúde Pública, v. 22, n. 3, p. 587-595, 2006.

BRIAN, P; CARRASCO, C; SPEISKY, H. Boldine and its antioxidant or healthpromoting properties. Chemico-Biological Interactions, v. 159, n.1, p.1-17, 2006.

CAMARGO, M.T.L.A. Contribuição etnofarmacobotânica ao estudo de Petiveria alliacea L. - Phytolacaceae - ("amansa - senhor") e a atividade hipoglicemiante relacionada a transtornos mentais. Dominguezia, v. 23, n. 1, p. 21-27. 2007.

Consórcio Intermunicipal de Saúde do Setentrião Paranaense (CISAMUSEP). História. 2014. Disponível em: <http://www.cisamusep.org.br/historia> Acesso em: 01 Dez. 2014.

DI STASI, L. C.; HIRUMA-LIMA, C. A.. Plantas medicinais na Amazônia e na Mata Atlântica. 2 ed. rev. e amp. São Paulo: Unesp, p. 169. 2002.

GIELEN, K.; GOOSSENS, A. Occupational allergic contact dermatitis from drugs in healthcare workers. Contact Dermatitis, v.45, n. 5, p. 273-279, 2001.

GUEDES R.C.M., NOGUEIRA N.G.P., FUSCO-ALMEIDA A.M., SOUZA C.R.F.; OLIVEIRA W.P. Atividade Antimicrobiana de Extratos Brutos de Petiveria alliacea L. Latin American Journal of Pharmacy, v.28, n. 4, p.520-524, 2009.

JUNIOR, R. G. O.; de LAVOUR, E. M.; de OLIVEIRA, M. R.; de SOUZA, E. V.; da SILVA, M. A.; da SILVA, M. T. N. M.; NUNES, L. M. N. Revista Eletrônica de Farmácia, v. IX, n.3, p.16 - 28, 2012.

KUBEC, R.; MUSAH, R.A. Substitutted cysteine derivatives and thiosulfinate formation in Petiveria alliacea - part II. Phytochemistry, v. 61, n. 6, p. 675-80. 2002. 
LIMA I.O., OLIVEIRA, R.A.G., LIMA, E.O., FARIAS, N.M.P., DE SOUZA, E.L. Atividade antifúngica de óleos essenciais sobre espécies de Candida. Revista Brasileira Farmacognosia , v.16, n. 2, p.197-201, 2006.

LORENZI, H; MATOS, F.J.A. Plantas medicinais no Brasil: nativas e exóticas cultivadas. São Paulo, Ed. Nova Odessa: Instituto Plantarum, 512p. 2002.

MEJÍA-DOLORES, J.W.; MENDOZA-QUISPE, D.E.; MORENO-RUMAY, E.L.; GONZALES-MEDINA, C.A.; REMUZGO-ARTEZANO, F.; MORALES-IPANAQUÉ, L.A. Efecto neurotóxico del extracto acuoso de boldo (Peumus boldus) en un modelo animal. Revista Peruana Medicina Experimental y Salud Publica, v.31, n.1, p.6268. 2014.

MONZÓN, S.; LEZAUN, A.; SÁENZ, D.; MARQINEZ, Z.; BERNEDO, N.; URIEL, O.; COLAS, C.; DUCE, F. Anaphylaxis to boldo infusion, a herbal remedy. Allergy, v. 59, n.9, p.1019-1020, 2004.

NDHLALA, A.R.; NCUBE, B.; OKEM, A.; MULAUDZI, R. B.; VAN STADEN, J. Toxicology of some important medicinal plants in southern Africa. Food and Chemical Toxicology, v.62, p. 609-621, 2013.

OCHOA, C.; GRANDA, C.; CHAPONAN, M.; BORJA, R.; BORJAS, P.; ORTIZ, J.; UGAZ, G.; PUERTA, E.; PUCUTAY, M. Efecto Protector de Peumus boldus en ratas con toxicidad hepática inducida por Paracetamol. Ciencia e Investigación Médica Estudiantil Latinoamericana, v.13, n.1, p.20-25, 2008.

OLIVEIRA, R. B; GODOY, S. A. P.; COSTA, F. B. Plantas tóxicas: conhecimento para a prevenção de acidentes. Ribeirão Preto: Holos. 64p. 2006.

OTAIZA, R. G.; ARZOLA, J. C.; RODRíGUEZ, M. C. Estudió etnobotánico de especies toxicas, ornamentales y medicinales de uso popular, presentes en el Jardín de Plantas Medicinales " Dr.Luis Ruiz Terán" de la Facultad de Farmacia y Bioanálisis de la Universidad de Los Andes. Boletín Antropológico, v. 24, n. 68, p 463-481, 2006.

PEACOK, B.M.; CRESPO, M.F.S.; RIVAS, C.A.B.; PÉREZ, L. Intoxicaciones por plantas tóxicas atendidas desde un servicio de información toxicológica. Revista Cubana de Pantas Medicinales, v.14, n.2, 2009.

PINILLOS, M. A; GÓMEZ, J; ELIZALDE, J. DUENAS, A.. Intoxicacion por alimentos, plantas y setas. Anales Sin San Navarra, v.26, n.1, p. 243-263, 2003.

RODRIGUES, A. C. C.; GUEDES, M. L. S.. Utilização de plantas medicinais no Povoado Sapucaia, Cruz das Almas-Bahia. Revista Brasileira Plantas Medicinais, v.8, n.2, p. 1-7, 2006.

RUIZ, A.L.T.G; TAFFARELLO, D.; SOUZA, V.H.S.; CARVALHO, J. E. Farmacologia e toxicologia de Peumus boldus e Baccharis genistelloides. Revista Brasileira de

Farmacognosia, v.18, n.2, p.295-300, 2008. 
SIMÕES, C. M. O; SCHENKEL, E. P.; GOSMANN, G.; MELLO, J. C. P.; MENTZ, L. A.; PETRVICK, P. R. Farmacognosia: da planta ao medicamento. 6 ed. Porto Alegre:Editora da UFRGS/Editora da UFSC, 2010.

SINITOX - Fundação Oswaldo Cruz/Centro de Informação Científica e Tecnológica/Sistema Nacional de Informações Tóxico-Farmacológicas (FIOCRUZ/CICT/SINITOX). Plantas tóxicas no Brasil. 2009. Disponível em $<$ http://www.fiocruz.br/sinitox novo/cgi/cgilua.exe/sys/start.htm?sid=313>. Acesso em: 01 Jan. 2015.

SINITOX - Fundação Oswaldo Cruz/Centro de Informação Científica e Tecnológica/Sistema Nacional de Informações Tóxico-Farmacológicas (FIOCRUZ/CICT/SINITOX). Casos registrados de intoxicação e/ou envenenamento. 2015.2 Disponível em: http://www.fiocruz.br/sinitox/cgi/cgilua.exe/sys/start.htm?sid=411>. Acesso em: 06 Jul. 2015.

VASCONCELOS, J.; VIEIRA, J. G. de P.; VIEIRA, E. P. de P. Plantas Tóxicas: Conhecer para Prevenir. Revista Científica da UFPA, v. 7, n. 1, p. 1-6, 2009.

VILA, R.; VALENZUELA, L.; BELLO, H.; CANIGUERAL, S.; MONTES, M.; ADZET, T. Composition and antimicrobial activity of the essential oil of Peumus boldus leaves. Planta Medica, v.65, n. 2, p.178-179, 1999.

YU, B.; COOK, C.; SANTANAM, N. The aporphine alkaloid boldine induces adiponectin expression ando regulation in $3 \mathrm{~T} 3-\mathrm{L} 1$ cells. Journal of Medicinal Food, v.12, n.5, p.1074-83. 2009. 